\title{
Cuidado ao idoso deprimido e institucionalizado à luz da Complexidade
}

\section{Care to the depressed senior and institutionalized to the light of the Complexity}

\author{
Cuidado del anciano depresivo e institucionalizado a luz de la Complejidad
}

\author{
Cenir Gonçalves Tier', Valéria Lerch Lunardi", Silvana Sidney Costa Santos"II
}

\section{RESUMO}

Instituições de Longa Permanência para Idosos (ILPI) são estabelecimentos para atendimento integral, institucionalizando pessoas de 60 anos e mais, dependentes/ou não, que não dispõem de condições para permanecer com a família ou/em seu domicílio. A institucionalização pode desencadear doenças, dentre elas a depressão. Foi objetivo deste artigo de atualização fazer uma reflexão sobre a relação do cuidado ao idoso deprimido e residente em ILPI com três princípios da Complexidade segundo Edgar Morin. Primeiro, se reflete sobre a complexidade; depois, se relaciona o cuidado ao idoso deprimido e residente em ILPI, com os princípios dialógico, recursivo e hologramático. Percebeu-se que a Complexidade pode sustentar teoricamente, não só a compreensão do fenômeno depressão no idoso residente em uma ILPI, como também inspirar o modo de cuidar que direcione aos trabalhadores da saúde, que atuam junto à pessoa idosa, a abordarem o idoso de forma mais global. Para tanto deve ser utilizada a Avaliação Multidimensional do Idoso, que analisa as várias dimensões da pessoa idosa, contribuindo no desenvolvimento de um plano de cuidado/tratamento e acompanhamento multidisciplinar e interdisciplinar adequados. A Complexidade vem sendo utilizada, por enfermeiros, para entender melhor a enfermagem e prestar cuidado mais adequado ao ser humano.

Palavras chave: Enfermagem geriátrica; Saúde do idoso institucionalizado; Assistência à saúde.

\section{ABSTRACT}

Institutions of Long Stay for the Elderly (ILPI) are establishments for integral service, institutionalizing 60 year-old people and plus, dependents/or no, that don't have conditions to stay with the family or in their home. The institucionalization can unchain diseases, among them the depression. The objective of this updating article was to do a reflection about the relationship of the care to the depressed senior and resident in ILPI with three beginnings of the Complexity according to Edgar Morin. First, we make reflections about the complexity; then, we link the care to the depressed senior and resident in ILPI, with the beginnings: dialogical, recursive and hologrammar. We realised that the Complexity can sustain theoretically, not only the understanding of the phenomenon depression in the resident senior in an ILPI, as well as to inspire the way of taking care that it addresses the workers of the health, that act the senior person close to, they approach the senior in a more global way. For so much the Multidimensional Senior's Evaluation should be used, it evaluates the senior person's several dimensions, contributing in the development of a care/treatment plan and multiact attendance and interdisciplinary appropriate. The Complexity has been used, for nurses, to understand the nursing better and to render more appropriate care to the human being.

Key words: Geriatric Nursing; Health of Institutionalized Elderly; Delivery of Health Care.

\section{RESUMEN}

Las instituciones de larga estancia para el adulto mayor (ILPI) son los establecimientos para atención integral, institucionalizando las personas de 60 años y más, dependientes o no,

Enfermeira. Mestre em Enfermagem pela Fundação Universidade Federal do Rio Grande (FURG). Membro do Grupo de Estudo e Pesquisa em Gerontogeriatria, Enfermagem/Saúde e Educação (GEP-GERON/FURG/CNPq). E-mail:cgtier@hotmail.com.

"Enfermeira. Doutora em Enfermagem. Pesquisadora do CNPq. Professora do Departamento de Enfermagem da FURG. Rio Grande/RS. Líder do Núcleo de Estudos e Pesquisas em Saúde (NEPES/FURG/CNPq). Email:vlunardi@terra.com.br

III Enfermeira. Doutora em Enfermagem. Professora do Departamento de Enfermagem da FURG. Líder do GEPGERON/FURG/CNPq. Membro do Grupo de Estudo e Pesquisa da Complexidade (GEC/FURG/CNPq) e do GRUPEC (UNINOVE/SP). Rio Grande/RS.

Email:silvanasidney@terra.com.br 
que no tienen condiciones para permanecer con la familia o/en su hogar. La institucionalización puede desencadenar enfermedades, entre ellas la depresión. Fue objetivo de esto artículo de actualización hacer una reflexión acerca de la relación del cuidado al anciano deprimido y residente en ILEA con tres principios de la Complejidad, segundo Edgar Morin. En primer lugar, si hace reflexiones sobre el complejidad, después, si refiere a la atención de ancianos con depresión y residente en ILEA, con los principios dialógico, recursivo y hologramático. Se observó que la teoría de la complejidad puede sustentar teóricamente, no sólo la comprensión del fenómeno de la depresión en los ancianos que viven en una ILEA, sino también inspirar el modo de atención que direccione los

\section{NTRODUÇÃO}

O envelhecimento é complexo, com implicações para o ser humano que o vivencia e para sua família/sociedade. Como processo irreversível o envelhecimento a que todos os seres vivos estão sujeitos necessita ser mais bem compreendido, principalmente, no momento em que o Brasil enfrenta um crescente número de pessoas idosas, modificando sua pirâmide populacional ${ }^{(1)}$.

Frente a esse novo contingente populacional, verifica-se a necessidade de formação/capacitação dos trabalhadores de saúde e de estruturação dos serviços para melhor cuidá-las e, principalmente, para pesquisar com e sobre as pessoas idosas.

O envelhecimento necessita ser compreendido de modo interdisciplinar; precisa ser percebido no próprio ser humano e em uma dimensão ecológica, considerando questões como gênero, geração, classe social e etnia. O envelhecimento habitualmente apresenta-se como um importante indicador no processo saúde e doença(2) ${ }^{(2)}$ O processo de envelhecimento é resultado de mudanças ligadas ao tempo em decorrência da deterioração de um organismo. Tais mudanças são próprias a todos os membros de uma espécie, sendo o tempo responsável por se tornarem cada vez menos capazes para o enfrentamento do estresse do ambiente ${ }^{(3)}$. De modo geral o envelhecimento é visto ainda como um processo evolutivo e inevitável na vida dos seres humanos. Porém, muitas vezes o trabajadores de la salud, que trabajan junto a la persona anciana, a abordar el ancianos de manera más global. Para tanto debe utilizarse la Evaluación Multidimensional del Anciano, que evalúa las diversas dimensiones de la persona anciana, contribuyendo en el desarrollo de un plan de atención / tratamiento y acompañamiento multidisciplinario e interdisciplinario adecuado. La complejidad ha sido utilizada por los enfermeros, para comprender mejor la enfermería y proporcionar la atención más adecuada para el ser humano.

Palabras clave: Enfermería geriátrica; Salud del anciano institucionalizado; Prestación de atención de salud.

envelhecimento é associado a doença e incapacidade. Assim, a velhice não é percebida socialmente como mais uma fase da vida humana ${ }^{(3)}$.

Atrelado ainda a um estigma de inutilidade, as pessoas idosas têm de enfrentar as perdas emocionais que se intensificam no final da vida. São os filhos que se casam, parentes que morrem, amigos que se vão e, o mais dolorido, o afastamento ou a perda do esposo/esposa ${ }^{(4)}$. Por outro lado as famílias vão encontrando dificuldades em cuidar dos seus idosos, sendo uma realidade cada vez mais presente, mesmo que questionada por alguns. Assim, se torna necessária a existência da Instituição de Longa Permanência para Idosos (ILPI), antes denominada asilo ou conhecidas por abrigo, lar, casa de repouso clínica geriátrica e ancionato.

A ILPI é um estabelecimento para atendimento integral institucional, acolhendo pessoas de 60 anos e mais, dependentes ou não, que não dispõem de condições para permanecer com a família e/ou em seu domicílio ${ }^{(5)}$.

Fatores como limitações físicas, dependência funcional verificados nos idosos residentes em ILPI, associam-se ao isolamento e à negação da percepção de um ambiente que pode não lhes ser agradável, afetando profundamente seus sentimentos, contribuindo para o desenvolvimento de doenças não apenas físicas como também psicológicas ${ }^{(6)}$. Este fato se agrava porque a população idosa constitui 
uma faixa etária mais propensa a ter problemas de saúde, principalmente a presença de Doenças Crônicas Não-Transmissíveis (DCNT), que podem apresentar baixa letalidade (possibilidade de morte) e alto grau de incapacitação(2).

O processo de envelhecimento apresenta variações que são constituídas nos diferentes grupos sociais, de acordo com a visão de mundo compartilhada em práticas, crenças e valores. A visão clínico-biológica não contempla essa perspectiva, ao diferenciar as fases da vida em infância, juventude, adulta e velhice, seguindo uma ordenação linear cronológica de transformações do corpo ${ }^{(3)}$. Nesta seqüência, pode ocorrer uma progressiva deterioração das funções vitais, em que o envelhecimento é caracterizado por um período de falência gradativa dos órgãos, podendo agregar características como tristeza, abandono, desrespeito, exclusão dos meios de produção, carências afetivas e materiais ${ }^{(6)}$.

Muitos foram os fatores que impediram o progresso do conhecimento sobre o envelhecimento, sendo o mais importante, sem dúvida, a constância com que o envelhecimento natural foi erroneamente caracterizado como um estado de doença, o que estimulou muito mais as tentativas de combatê-lo do que de entendê-lo ${ }^{(3)}$.

Embora, no Brasil, algumas políticas venham sendo implementadas para valorizar as pessoas idosas como cidadãs, é fato que elas enfrentam várias situações de exclusão, pois a cultura brasileira associa velhice à dependência física, às doenças, à inutilidade ${ }^{(2)}$. Como já referido o processo de envelhecimento poderá trazer limitações e aparecimento de doenças próprias da faixa etária acima de 60 anos e mais, como a Doença de Alzheimer, a depressão, dentre outras.

A depressão é a principal doença mental das pessoas idosas, não só por sua grande freqüência, mas também pelas implicações que acarreta sobre todo o organismo. É ainda uma doença que, além de ocasionar repercussões sociais e individuais, afeta o convívio social, impossibilitando uma rotina de vida satisfatória, apresentando, desta forma, risco inerente de morbidade e cronicidade ${ }^{(6)}$.
Refletindo sobre o cuidado à pessoa idosa deprimida e residente em uma Instituição de Longa Permanência para Idosos, é possível visualizar pontos precursores de uma mudança de cuidar na ILPI, já que até há pouco tempo quase nada era realizado com estes seres humanos e aqueles asilos funcionavam como depósitos de velhos. Atualmente prioriza-se a avaliação denominada multidimensional em idosos e, por meio dela, se podem identificar limitações e/ou doenças, entre elas a depressão, o mais precocemente possível, o que vem acarretando possíveis modificações no cuidar de idosos deprimidos institucionalizados.

É importante que o idoso residente em ILPI seja objeto de avaliação multidimensional, sendo esta definida como um processo diagnóstico envolvendo várias dimensões, usualmente multidisciplinar e interdisciplinar, capaz de avaliar a capacidade biológica, psicossocial e funcional da pessoa idosa, objetivando desenvolver um plano de tratamento e acompanhamento adequados ${ }^{(7)}$. Nessa perspectiva sugere-se a aplicação da Avaliação Multidimensional do Idoso, que pode ser considerada uma nova maneira de lidar com os idosos residentes em ILPI, principalmente os que apresentam distúrbios afetivos e esse novo olhar poderá ser melhor percebido por meio da utilização da Complexidade de Edgar Morin ${ }^{(8,11)}$.

Nesse artigo de atualização procurou-se apresentar esse novo olhar, realizando-se inicialmente uma breve contextualização acerca da complexidade, segundo Edgar Morin (8,11); elaborando-se em seguida uma interconexão entre cuidado ao idoso deprimido e residente em uma ILPI e os princípios dialógico, o recursivo e o hologramático da Complexidade Moriniana $^{(8,11)}$.

Assim, este artigo teve como objetivo fazer uma reflexão sobre a relação do cuidado ao idoso deprimido e residente em Instituição de Longa Permanência para Idoso (ILPI) com três princípios da Complexidade segundo Edgar Morin.

\section{A COMPLEXIDADE SEGUNDO EDGAR MORIN}

Neste século tem-se urgência de pensar novas alternativas diante do mundo, das relações e das organizações. São os 
pensamentos e ações que determinam a cultura e, ao mesmo tempo, são determinados por ela. As sociedades, em sua diversidade múltipla, ditam regras e normas que são aceitas e incorporadas pelas comunidades, objetivando, cada vez mais, adequar e unificar procedimentos e critérios, que não apenas distinguem os povos, mas, sobretudo, aproximam os seres humanos membros de um $\operatorname{grupo}^{(8-9)}$.

A vida atualmente direciona a possibilidade de se refletir sobre a necessidade de se adotar novos comportamentos e posturas que são influenciados pelo modo de pensar. Assim, os pensamentos determinam as práticas que se estabelecem e se desenvolvem nas sociedades. Com freqüência, a urgência e as rápidas mudanças nas diversas áreas do saber indicam que a aprendizagem dos seres humanos ocorre em toda parte e em todos os tempos ${ }^{(8,10)}$.

A Complexidade é vista como um tipo de pensamento que não separa, mas une e busca as relações necessárias e interdependentes de todos os aspectos da vida humana. É um pensamento integrador dos diferentes modos de pensar, opondo-se aos mecanismos reducionistas, disjuntivos e simplificadores. Por meio do pensamento complexo são consideradas todas as influências recebidas, externas e internas, e ainda a incerteza e a contradição, sem deixar de conviver com a solidariedade dos fenômenos existentes ${ }^{(11-12)}$. Portanto, a Complexidade é entendida como um pensamento sem certezas e sem verdades científicas, que considera a diversidade e a incompatibilidade de idéias, percepções e crenças, integrando-as à sua complementaridade. Tem a consciência de nunca ter certeza e desenvolve a capacidade de transpor a ambigüidade e a incerteza ${ }^{(11)}$.

A Complexidade leva o ser humano ao paradoxo do uno e do múltiplo e à convivência com a ambivalência, competindo a este ser humano, por meio do conhecimento, interpretar os aspectos ambíguos da realidade, sem desconsiderar sua multidimensionalidade, porque também as unidades complexas são multidimensionais ${ }^{(11)}$. Enfim, a Complexidade é uma maneira de entender o mundo, integrando relações de co-existência entre os seres vivos e não vivos, integrando conceitos de ordem e desordem, uno e diverso, estabilidade e mudança e, principalmente, a noção de incerteza ${ }^{(8)}$.

Os enfermeiros começam a inserir a complexidade nas ações da Enfermagem por meio de reflexões e propostas de mudanças quanto à organização/administração $0^{(9,13)}$,

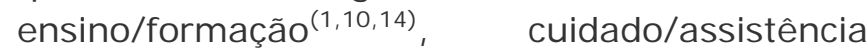
(12,15), utilizando-se de categorias bases da complexidade (incerteza, complementaridade, dialogicidade, interdisciplinaridade e transdisciplinaridade, e outras) ou de seus princípios.

\section{CUI DADO AO IDOSO INSTITUCI ONALIZADO DEPRIMIDO E A COMPLEXI DADE MORINI ANA}

O cuidado à pessoa idosa deprimida, principalmente a que reside em Instituição de Longa permanência para Idosos, neste texto se relaciona à Complexidade segundo Edgar Morin ${ }^{(8,11)}$, fazendo um paralelo com três princípios: dialógico, recursivo e hologramático.

O princípio dialógico objetiva unir noções antagônicas, juntar o que aparentemente deveria se manter separado, criando processos organizadores e complexos ${ }^{(8,11)}$. No que se refere à pessoa idosa e deprimida, percebe-se que ela pode apresentar sintomas de depressão mascarados e muitas vezes confundidos, pelos trabalhadores de saúde que o atendem, como características próprias do processo fisiológico de envelhecimento, dificultando a obtenção de um diagnóstico preciso e rápido. Comumente surge a dúvida: a pessoa idosa apresenta depressão ou estar com manifestações próprias do processo de envelhecimento? Assim, a dialogicidade apresenta-se na presença da depressão ou na sua ausência; pela proximidade dessas questões no idoso. A presença/ausência de depressão, apesar de serem questões antagônicas, aponta a necessidade de capacitação dos trabalhadores de saúde para realizarem, de forma adequada, esta identificação de sintomas da depressão, por meio da Avaliação Multidimensional do Idoso.

Por isso, a necessidade premente da inclusão de conteúdos, ou matérias, sejam optativas ou obrigatórias, nos Projetos 
Tier CG, Lunardi VLL, Santos SSC. Cuidado ao idoso deprimido e institucionalizado à luz da Complexidade. Revista Eletrônica de Enfermagem [Internet]. 2008;10(2):530-536. Available from: http://www. fen.ufg.br/revista/v10/n2/v10n2a24.htm

Pedagógicos dos Cursos (PPC) da saúde e principalmente nos cursos de Enfermagem, que reflitam/discutam o processo de envelhecimento, cuidado ao ser humano idoso, perspectivas de mudanças esperadas na fase da velhice. Esta é uma responsabilidade dos órgãos formadores de recursos humanos em saúde.

Já o princípio recursivo considera que os produtos e efeitos são ao mesmo tempo causas e produtores daquilo que os produziu, discordando da linearidade dos fatos, promovendo a criação de novos sistemas, nos quais os efeitos retroagem sobre as causas desencadeadoras ${ }^{(8,11)}$. Neste princípio, verificase que uma causa não está diretamente ligada à sua conseqüência, e assim, percebe-se que nenhum fator explica satisfatoriamente a gênese de sintomas depressivos ou da instalação da depressão na pessoa idosa. Em proporções variáveis, da soma de diversos fatores psicossociais resulta um grupo heterogêneo de sinais e sintomas depressivos encontrados nos idosos, principalmente nos que residem em $\mathrm{ILPI}^{(6)}$.

Diversos fatores podem ser considerados predisponentes ou desencadeadores de quadros depressivos na pessoa idosa. Um mesmo fator pode ser considerado predisponente ou desencadeador, dependendo da história de cada idoso. Os sintomas podem surgir ainda devido a fatores genéticos, neurobiológicos, psicossociais, alterações psicológicas, sociais, econômicas que poderão ou não está associados ao processo de envelhecimento ${ }^{(6)}$. Reações à perda de um parente exercem importante papel como fator desencadeante de quadros depressivos em pessoas idosas. Outros elementos como diminuição do suporte social; isolamento social; insatisfação com o suporte proporcionado por familiares; diminuição da saúde física, do nível de atividade, da independência; grande incidência de doenças; prática de polifarmacia; presença de dor crônica; entre outros fatores, podem contribuir para o aumento de risco de desenvolvimento de depressão nos idosos ${ }^{(6)}$.

Considera-se que o idoso residente em ILPI está exposto a um ambiente que pode propiciar o desenvolvimento de sintomas depressivos, como ele estar afastado de seu ambiente familiar, sendo levado a conviver com pessoas estranhas e a ter que se adaptar as novas rotinas. Na maioria das vezes, estar isolado da atualidade cultural, além de estar experimentando algumas situações incômodas, como o abandono por parte dos seus familiares, dependência de horários e rotinas da ILPI e outras $^{(6)}$.

Por fim, o princípio hologramático defende que não apenas a parte está no todo, mas que o todo está, de certa forma, na parte. Por meio deste princípio enriquece-se o conhecimento das partes pelo todo e do todo pelas partes, num movimento produtor de conhecimentos $^{(8,11)}$. Correlacionando este princípio à depressão no idoso institucionalizado, percebe-se que a depressão não é um problema apenas da pessoa idosa, mas também de sua família/comunidade/ILPI em que ele reside, ou seja, depende também do contexto em que ele está inserido. O idoso pode se tornar uma pessoa deprimida em decorrência do descaso de sua família e/ou do grupo de que ele faz parte, mas, por outro lado, a família/grupo também sofre com o comportamento do idoso.

Portanto, a interação da pessoa idosa (parte) e da família/grupo/ILPI (todo) necessita ser adequada ao atendimento das necessidades específicas tanto do idoso institucionalizado como da família ou de outros idosos e trabalhadores/cuidadores, no caso da ILPI.

Após refletir e relacionar alguns princípios da Complexidade, de Edgar Morin e a presença de depressão em idosos residentes em ILPI, volta-se a enfatizar a importância da Avaliação Multidimensional do Idoso, principalmente para o institucionalizado. Por meio desta avaliação multidimensional, global ou geriátrica ampliada pode-se realizar uma melhor análise dos problemas da pessoa idosa. Esta tecnologia constitui um instrumento que não negligencia as partes para compreender o todo, avaliando a pessoa idosa individual e globalmente, possibilitando-lhe um cuidado mais humanizado, pois parte de uma equipe multidisciplinar com vistas à atuação interdisciplinar, o que tenderá a contribuir para uma melhora na qualidade de vida do idoso institucionalizado.

Pelas relações estabelecidas entre a depressão em idosos residentes em Instituição 
de Longa Permanência para Idosos e alguns princípios de Edgar Morin ${ }^{(8,11)}$, percebeu-se que a Complexidade pode sustentar teoricamente, não apenas a compreensão do fenômeno depressão no idoso residente em ILPI, como ainda inspirar o modo de cuidar mais adequado. Esse cuidar surge mediante um novo olhar, que direciona os trabalhadores da saúde, principalmente os enfermeiros, a verem a pessoa idosa de forma mais global e atenta ao que ocorre com o processo de envelhecimento e suas alterações, o que pode ser favorecido pelo uso da Avaliação Multidimensional do Idoso.

\section{CONSI DERAÇÕES FI NAIS}

Os fenômenos relacionados ao processo de envelhecimento e a fase da velhice, além da determinação de quem seja idoso, muitas vezes são considerados com referência às restritas modificações que ocorrem no corpo, na dimensão física.

Faz-se necessário entender que, ao longo dos anos, são processadas mudanças na forma de pensar, de sentir e de agir dos seres humanos que cursam esta etapa do processo de viver. Várias dimensões: biológica, psicológica, social, espiritual dentre outras, necessitam ser consideradas no cuidado à pessoa idosa, possibilitando a aproximação de um conceito que a abranja e que a perceba como ser multidimensional e complexo.

A pessoa idosa deprimida faz parte de um contexto complexo e necessita ser vista em suas especificidades e multidimensionalidade, o que pode ser ignorado por muitos trabalhadores da saúde, reforçando a importância de seu preparo para cuidar dos idosos, tanto na vida acadêmica, como profissional, por meio da educação permanente.

Defendeu-se a importância de uma mudança de cuidado/tratamento direcionado à aplicação da avaliação multidimensional para o idoso, que reside em Instituição de Longa Permanência, de modo a poder-se identificar e tratar a depressão, tanto mais precocemente quanto o mais adequadamente possível, além de buscar alternativas para contribuir na melhoria da qualidade de vida da pessoa idosa institucionalizada.

Deste modo há a necessidade de que os cursos/escolas da área da saúde, principalmente da Enfermagem, incluam este ensino/preparo em seu Projeto Pedagógico de Curso (PPC), habilitando e capacitando os futuros trabalhadores a identificarem problemas nas pessoas idosas, o mais precoce possível e, assim, contribuírem para que elas tenham uma melhor qualidade de vida.

\section{REFERÊNCI AS}

1. Santos SSC. O ensino da enfermagem gerontogeriátrica e a complexidade. Revista da Escola de Enfermagem da USP. 2006; 40(2): 228-35.

2. Brêtas AC. Políticas públicas de saúde para o envelhecimento: a ousadia de cumprir a lei. Revista de Ciências da Saúde da UFSC. 2005; 24(1/2): 51-55.

3. Tier CG, Soares NV, Fontana RT. Refletindo sobre idosos institucionalizados. Revista Brasileira de Enfermagem. 2004;57(3):332-35.

4. Lundh U, Sandedberg J, Nolan M. 'I don't have any other choise': spouses' experiences of placing a partner in a care home for older people in Sweden. Journal of advanced nursing. 2000; 32(5): 1178-1186.

5. Sociedade Brasileira de Geriatria e Gerontologia; Regional São Paulo [Internet]. São Paulo: Sociedade Brasileira de Geriatria e Gerontologia (BR) [cited $2007 \mathrm{dez}$ 15]. CARTA ABERTA SOBRE ILPIs. Available from: http://www. sbgg-sp.com. br/noticias/noticia006. htm.

6. Andrade AC, Lima FRA, Silva LFA, Santos SSC. Depressão em idosos de uma Instituição de Longa Permanência: proposta de ações de enfermagem. Revista Gaúcha de Enfermagem. 2005; 26(1): 57-66.

7. Santos SSC, Feliciani AM, Silva BT. Perfil de idosos residentes em Instituição de Longa Permanência: proposta de ações de enfermagem/saúde. Revista da Rede de Enfermagem do Nordeste - RENE. 2007; 8(3): 26-33.

8. Morin E. A Cabeça bem-feita: repensar a reforma, reformar o pensamento. $2^{\underline{a}}$ ed. Rio de Janeiro: Bertrand Brasil; 2000.

9. Erdmann AL, Souza FGM, Backes DS, Mello ALSF. Construindo um modelo de sistema de cuidados. Revista Acta Paulista de Enfermagem. 2007; 20(2): 180-5.

10. Silva AL, Camillo SO. A educação em enfermagem à luz do paradigma da 
complexidade. Revista da Escola de

Enfermagem da USP. 2007; 41(3):403-10.

11. Morin E. Ciência com consciência. 8a ed. Rio de Janeiro: Bertrand Brasil; 2005.

12. Terra MG, Camponogara S, Silva LC, Girondi JBR, Nascimento K, Radüz V. et al. O significado de cuidar no contexto do pensamento complexo: novas possibilidades para a enfermagem. Rev Texto e Contexto em Enfermagem. 2006; 15(esp): 164-9.

13. Erdmann AL, Andrade SR, Mello ALSF, Meirelles BHS. Gestão das práticas de saúde na perspectiva do cuidado complexo. Rev Texto e Contexto em Enfermagem. 2006; 15(3): 483-91.

14. Santos SSC. Educação em Enfermagem e a complexidade. Revista Contexto e Educação da UNIJUí. 2005; 20(73/74): 103-17.

15. Lopes FL, Szewczyk MSC, Lunardi VL, Santos SCS. SAE como um novo fazer na atividade cuidativa da enfermagem com base na complexidade de Edgar Morin. Cogitare Enfermagem. 2007; 12(6): 109-13.

Artigo recebido em 27.03.08

Aprovado para publicação em 30.06.08 